\title{
Correction to: Exposing the probabilistic causal structure of discrimination
}

\author{
Francesco Bonchi ${ }^{1} \cdot$ Sara Hajian ${ }^{2} \cdot$ Bud Mishra $^{3}$. Daniele Ramazzotti ${ }^{4}$
}

Published online: 8 April 2019

C) Springer Nature Switzerland AG 2019

\section{Correction to: Int J Data Sci Anal (2017) 3:1-21 https://doi.org/10.1007/s41060-016-0040-z}

In the original publication of the paper, the in-text citation for reference 33 was published in the incorrect place. The citation currently appears in the following sentence:

"Based on a detailed empirical analysis of Kleinberg [33] and her coauthors ...."

However, the correct position of the citation should be as follows:

"We refer the interested reader to [33] and citations within."

In addition to this, the use of the story of the Sneetches as an analogy to help illustrate causal discrimination was originally sourced from Blank RM, Dabady M, Citro CF. Measuring racial discrimination. Natl Academy Pr; 2004. A reference to the book by Blank et al. (2004) was included in the text, but it should have been included in multiple instances in Section 1.2.

Publisher's Note Springer Nature remains neutral with regard to jurisdictional claims in published maps and institutional affiliations.

The original article can be found online at https://doi.org/10.1007/ s41060-016-0040-z.

Sara Hajian

sara.hajian@eurecat.org

Francesco Bonchi

francesco.bonchi@isi.it

Bud Mishra

mishra@nyu.edu

Daniele Ramazzotti

daniele.ramazzotti@disco.unimib.it

1 Algorithmic Data Analytics Lab, ISI Foundation, Turin, Italy

2 Eurecat-Technology Centre of Catalonia, Barcelona, Spain

3 New York University, New York, NY, USA

4 Milano-Bicocca University, Milan, Italy 